\title{
Cooperative Exploration for USAR Robots with Indirect Communication
}

\author{
V. A. Ziparo, Alexander Kleiner, L. Marchetti, A. Farinelli and D. Nardi
}

\section{Post Print}

N.B.: When citing this work, cite the original article.

Original Publication:

V. A. Ziparo, Alexander Kleiner, L. Marchetti, A. Farinelli and D. Nardi, Cooperative Exploration for USAR Robots with Indirect Communication, 2007, In Proc. of 6th IFAC Symposium on Intelligent Autonomous Vehicles (IAV).

Postprint available at: Linköping University Electronic Press

http://urn.kb.se/resolve?urn=urn:nbn:se:liu:diva-72571 


\title{
COOPERATIVE EXPLORATION FOR USAR ROBOTS WITH INDIRECT COMMUNICATION
}

\author{
Vittorio Amos Ziparo* Alexander Kleiner** \\ Alessandro Farinelli* Luca Marchetti* \\ Daniele Nardi * \\ * University of Rome "Sapienza", Via Salaria 113, 00198 \\ Rome, Italy, \{lastname\}@dis. uniroma1.it \\ ** University of Freiburg, Georges-Köhler-Allee 52, \\ D-79110 Freiburg, Germany, \\ kleiner@informatik .uni-freiburg.de
}

\begin{abstract}
To coordinate a team of robots for exploration is a challenging problem, particularly in unstructured areas, as for example post-disaster scenarios where direct communication is severely constrained. Furthermore, conventional methods of SLAM, e.g. those performing data association based on visual features, are doomed to fail due to bad visibility caused by smoke and fire. We use indirect communication (based on RFIDs), to share knowledge and use a gradient-like local search to direct robots towards interesting areas. To share a common frame of reference among robots we use a feature based SLAM approach (where features are RFIDs). The approach has been evaluated on a 3D simulation based on USARSim.
\end{abstract}

\section{INTRODUCTION}

Coordination of a team of robots for exploration is a challenging problem, particularly in large areas as for example the devastated area after a disaster. The problem for USAR (Urban Search and Rescue) scenario is generally harder, due to difficult operation conditions, i.e. bad visibility, caused by smoke and fire, and constraints for communication. In this paper, we tackle the core problems of coordinated exploration and multirobot Simultaneous Localization And Mapping (SLAM) for large teams of USAR robots. The exploration problem is solved using RFIDs for indirect communication, while the SLAM problem is solved by using RFIDs as features which are detectable in presence of low visibility via UHF antennas.

In this paper we show how RFID-based exploration (Ziparo et al., 2007) can be used when direct communication is severely restricted and the robots are equipped with noisy RFID sen- sors. Roughly, in RFID-based exploration teams of robots autonomously explore a disaster environment, while reducing significantly the size of the search space by utilizing RFID tags as coordination points. Each robot deploys autonomously RFID tags for building a network of reachable locations which represents a topological map of the environment annotated with metric positions for the features (i.e. RFID tags).

In order to estimate the true locations of RFIDs we utilize Extended Kalman Filter (EKF)-based SLAM with RFID landmarks as features, while achieving unambiguous data associations through the RFIDs transmitted ID. Maps generated by each robot can easily be merged at the end of the mission into a global topological map by performing the union of the local maps based on association of RFIDs. Moreover, the merged graph can be further corrected off-line so to ensure global consistency. For example, the method from $\mathrm{Lu}$ and Milios ( $\mathrm{Lu}$ and Milios, 1997), was success- 
fully adopted for optimizing RFID graphs within former work (Kleiner et al., 2006).

During execution, robots are coordinated via RFID chips and perform a local search which allows them to explore an environment with low computational overhead and communication constraints. In particular, the computational costs do not increase with the number of robots. The key idea is that the robots plan and explore based on a local view of the environment, which is maintained consistent through the use of indirect communication via RFID tags. The coordination mechanism, which is based on target selection and indirect communication, results in distributed local search.

Experiments reported in this work are evaluating the feasibility of RFID-technology based exploration and SLAM for a larger real robot experiment that will be conducted in the future. Therefore, they have been firstly carried out in the USARSim (Balakirsky et al., 2006) simulation environment, which serves as basis for the Virtual Robots competition at RoboCup. We modified the simulator in that it provides range readings of RFID tags with respect to a model for signal path attenuation. Our results show that the RFID tagbased exploration works for large robot teams, particularly if they have limited computational resources. Furthermore, the results show that the local map of each robot, i.e. the locations of RFIDs, can successfully be maintained consistent by updates from RFID range readings.

Methods for local exploration have already been successfully applied in the past (Balch and Arkin, 1994; Svennebring and Koenig, 2004). Transponders, such as RFIDs, have already been successfully utilized for localizing mobile robots (Hähnel et al., 2004; Bohn and Mattern, 2004) and emergency responders (Kantor et al., 2003; Miller et al., 2006). However, most of these methods require that the map of the environment is partially known, whereas the presented work describes a solution that performs RFID-based SLAM while exploring the environment. EKF-based SLAM has been extensively studied (Durrant-Whyte et $a l ., 1996)$. In connection with radio transmitters, the problem has mainly be addressed as "rangeonly" SLAM (Kehagias et al., 2006; Kurth et al., 2003; Kantor and Singh, 2002), since the bearing of the radio signal cannot always accurately be determined.

The remainder of this paper is structured as follows. In Section 2 we describe the system platform and in Section 3 the simulated RFID model. In Sections 4 and 5 the EKF-based SLAM and coordination methods based on RFIDs are discussed. Finally we present experimental results in Section 6 and draw some conclusions in Section 7.

\section{TEST PLATFORM}

The test platform utilized for experiments presented in this paper is based on a realistic model of the differentially steered Zerg robot, as depicted in Figure 1(a). The robot is equipped with a Hokuyo URG-X003 Laser Range Finder (LRF), and an Inertial Measurement Unit (IMU) from XSense providing measurements of the robot's orientation by the three Euler angles yaw, roll, and pitch. The

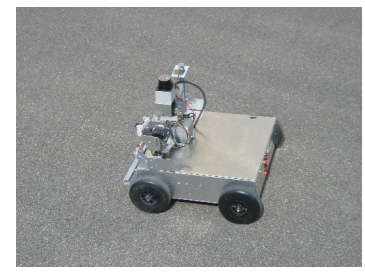

(a)

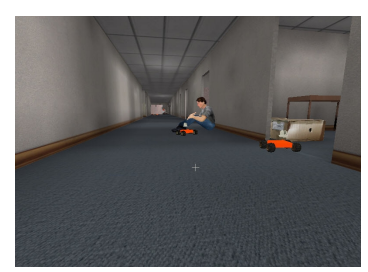

(b)
Fig. 1. The rescue robot Zerg (a) and the corresponding model (b) witin the USARSim simulation.

model (see Figure 1(b)) has been designed for the USARSim simulator developed at the University of Pittsburgh (Carpin et al., 2006; Balakirsky et al., 2006). USARSim allows a real-robot simulation of raw sensor data, which can directly be accessed via a TCP/IP interface, whereas sensors, such as odometry and LRF, can be simulated with the same parameters as they are found on real robots.

The simulated robot has been extendet by a model of a RFID system that we intend to utilize within a larger experiment on real robots in future work. The system consists of a CAEN A928EU UHF long range RFID reader and A918 UHF passive tags (CAEN, 2007). The reader operates at $869.525 \mathrm{M} \mathrm{Hz}$ with an output power of $630 \mathrm{~mW}$, and allows to detect 50 tags per second. The tags maintain an anti-collison protocoll, allowing the simultaneous detection of multiple RFIDs within range. The maximal range within they can be detected is 4 meters. Furthermore, we simulate two directional antennas, one facing into the driving direction of the robot, and the other one facing backwards into the opposite direction. Each antenna detects RFIDs within a field of view of approximately $60^{\circ}$.

\section{RFID SENSOR MODEL}

The Transceiver-Receiver (TR) separation, i.e. the distance between a detected RFID and the detector, can generally be estimated from the power of the signal. However, signal propagation in an indoor environment is perturbed by damping and reflections of the radio waves. Since these perturbations depend on the layout of the building, the construction material used, and the number and type of objects in the building, modeling the 
relation between signal path attenuation and TR separation is a challenging problem.

Seidel and Rapport introduced a model for path attenuation prediction that can also be parameterized for different building types and the number of floors between transceiver and receiver (Seidel and Rapport, 1992). This model has been evaluated for frequencies in the UHF domain, e.g. $914 \mathrm{MHz}$, which is also the frequency domain of the examined RFID system (see Section 2). RFID implementations operating in this domain are requiring a line of sight between the tag and the detector. This allows us to adopt a simpler version of the model from Seidel and Rapport, based on the assumption that RFID detections are not possible through walls. The utilized model relates the signal power $P$ to distance $d$ in the following way:

$$
P(d)[d B]=p\left(d_{0}\right)[d B]-10 n \log \frac{d}{d_{0}}+X_{\sigma}[d B],
$$

whereas $P\left(d_{0}\right)$ is the signal power at reference distance $d_{0}$ and $n$ denotes the mean path loss exponent that depends on the structure of the environment. Seidel and Rapport determined for transmissions at $914 \mathrm{MHz}$ a path loss of $31.7 \mathrm{~dB}$ at a reference distance of 1 meter. Furthermore, they determined for different building types characteristic values for $n$ and the standard deviation $\sigma$ of the signal. This model has been used with varying values for $n$ and $\sigma$ for evaluating the method described in the next section.

\section{RFID SLAM}

We utilize EKF-based SLAM (Durrant-Whyte et al., 1996) in order to compute simultaneously the locations of the robot and of the RFIDs. Hence, the pose of the robot and RFID locations are denoted by a single state vector. It is assumed that each RFID observation is composed of a range measurement $r$ and bearing measurement $\phi$, relative to the center of the receiving antenna. We compute range $r$ from the signal strength according to the model described in Section 3, while considering the spacial displacement of the specific antenna. From the arrangement of the antennas described in Section 2, we estimate the bearing $\phi$ of the detection within a $60^{\circ}$ cone. Furthermore, within each discrete time interval $t$, the traveled distance $d_{t}$ and the traveled angle $\alpha_{t}$ of the robot are measured by the wheel encoder odometry and the Inertial Measurement Unit (IMU), respectively. Given the pose of the robot by the vector $l=(x, y, \theta)^{T}$ with $3 \times 3$ covariance matrix $\Sigma_{l}$, and the locations of $n$ RFIDs by the vector $m=\left(x_{1}, y_{1}, x_{2}, y_{2}, \cdots, x_{n}, y_{n}\right)^{T}$ with $n \times n$ covariance matrix $\Sigma_{m}$, the single state vector $s$ is defined by:

$$
\begin{aligned}
s & =\left(\begin{array}{c}
l \\
m
\end{array}\right) \\
\Sigma_{s} & =\left(\begin{array}{cc}
\Sigma_{l} & \Sigma_{l m} \\
\Sigma_{m l} & \Sigma_{m}
\end{array}\right) .
\end{aligned}
$$

The single state vector is updated according to (Durrant-Whyte et al., 1996) from the input vector $u_{t}=\left(d_{t}, \alpha_{t}\right)$ with covariance matrix $\Sigma_{u}$ after the following model:

$$
l_{t}=F\left(l_{t-1}, u_{t}\right)=\left(\begin{array}{c}
x_{t-1}+\cos \left(\theta_{t-1}\right) d_{t} \\
y_{t-1}+\sin \left(\theta_{t-1}\right) d_{t} \\
\theta_{t-1}+\alpha_{t}
\end{array}\right)
$$

From a single RFID observation, given by the vector $z=(r, \phi)$ with $2 \times 2$ covariance matrix $\Sigma_{z}$, the state vector is updated as follows: If the RFID is unknown, i.e. has not been observed before, the state vector is augmented with the new observation. Otherwise, the observation is associated to the correct RFID by utilizing the unique ID number transmitted by the RFID. Note that this is a clear advantage of RFID technology if comparing the method to other techniques that perform data association by validation gating. Based on the current estimates of associated RFID $m_{i}=\left(x_{i}, y_{i}\right)$ and robot pose $l=(x, y, \theta)$, the observation is predicted by the following measurement function:

$$
H_{i}(s)=\left(\begin{array}{c}
\sqrt{\left(x_{i}-x\right)^{2}+\left(y_{i}-y\right)^{2}} \\
\tan ^{-1}\left(\frac{y_{i}-y}{x_{i}-x}\right)-\theta
\end{array}\right) .
$$

Finally, the state vector is updated from the observation according to (Durrant-Whyte et al., 1996).

\section{COORDINATED EXPLORATION AND PATH-PLANNING}

In this section we present a coordination mechanism which allows robots to explore an environment with low computational overhead and communication constraints. In particular, the computational costs do not increase with the number of robots. The key idea is that the robots plan their path and explore the area based on a local view of the environment, where consistency is maintained through the use of indirect communication, i.e. RFIDs.

\subsection{Navigation}

To efficiently and reactively navigate, each robot continuously plans paths based on its local information of the environment, which is maintained within an occupancy grid. This representation of the environment, for allowing fast computation, is limited in size. In particular, in our implementation, we restricted it to a four meter side square with forty $\mathrm{mm}$ resolution. The occupancy grid 
is shifted based on the odometry and continuously updated based on new scans. This avoids the accumulation of the odometry errors when moving, while having some memory of the past. We periodically select a target, as shown in the next subsection, and produce for it plans at high frequency. The continuous re-planning allows to reactively avoid newly perceived obstacles or unforeseen situations caused by errors in path following.

The path planning algorithm is based on A* (Russell and Norvig, 2003) with the Euclidean distance heuristic. We expand all the neighbors of a cell which are not obstructed (i.e. have an occupancy value lower than a given threshold). The cost function $c$ takes into account the length of the path and the vicinity of the obstacles to the path in the following way:

$$
c\left(s_{i+1}\right)=c\left(s_{i}\right)+d\left(s_{i+1}, s_{i}\right) *\left(1+\alpha * o c c\left(s_{i+1}\right)\right)
$$

where $\operatorname{occ}(s)$ is the current value of the occupancy grid in cell $s, d($.$) is the distance, and \alpha$ is a factor for varying the cost for passing nearby obstacles. Before planning, the grid is convoluted with a Gaussian kernel, which allows to keep robots as far as possible from obstacles.

While navigating in the environment, robots maintain a local RFIDs set (LRS), which contains all the perceived RFIDs which are in the range of the occupancy grid. On the basis of this information, new RFIDs are released in the environment by the robots in order to maintain a predefined density of the tags (in our implementation we take care of having the RFIDs at one meter distance from each other). Note that nowadays most of the RFID tags available on the market do implement an anti-collision protocol, and hence the detection of multiple RFIDs is possible at the same time.

\subsection{RFID-based Exploration}

The fundamental problem in the local exploration task is how to select targets for the path planner in order to minimize overlapping of explored areas. This involves: i) choosing a set of target locations $F=\left\{f_{j}\right\}$, ii) computing an utility value $u\left(f_{j}\right)$ for each target location $f_{j} \in F$ and iii) selecting the best target, based on the utility value, for which the path planner can find a trajectory.

We first identify a set of targets $F$ by extracting frontiers $F$ (Yamauchi, 1997) from the occupancy grid. We then order the set based on the following utility calculation:

$$
u\left(f_{j}\right)=-\gamma_{1} * \text { angle }\left(f_{j}\right)-\gamma_{2} * \operatorname{visited}\left(f_{j}\right)
$$

where $\operatorname{angle}\left(f_{j}\right)$ is a value which grows quadratically with the angle of the target with respect to the current heading of the robot. The angle factor can be thought as an inertial term, which prevents the robot from changing too often direction (which would result in an inefficient behavior). If the robot would have full memory of his perceptions (i.e. a global occupancy grid), the angle factor would be enough to allow a single robot to explore successfully. Due to the limitation of the occupancy grid, the robot will forget the areas previously explored and thus will possibly go through already explored ones.

In order to maintain a memory of the previously explored areas the robots store in the nearest RFID at writing distance poses $p$ from their trajectory (discretized at a lower resolution respect to the occupancy grid). The influence radius, e.g. the maximal distance in which poses are added, depends mainly on the memory capacity of the RFID tag. In our implementation, poses where added within a radius of 4 meters. Moreover, a value $\operatorname{count}(p)$ (Svennebring and Koenig, 2004) is associated with each pose $p$ in the memory of the RFID and is incremented by the robots every time the pose is added. These poses $p$ are then used to compute visited $\left(f_{j}\right)$ as

$$
\operatorname{visited}\left(f_{j}\right)=\sum_{r \in L R S} \sum_{p \in P_{r}}\left(1 / d\left(f_{j}, p\right)\right) * \operatorname{count}(p)
$$

, where $P_{r}$ is the set of poses associated with the RFID $r$. Finally, $\gamma_{1}$ and $\gamma_{2}$ are two parameters which control the trade-off between direction persistence and exploration. It is worth noticing that robots writing and reading from RFIDs, not only maintain memory of their own past but also of the other robots implementing a form of indirect communication. Thus, both multi-robot navigation and exploration, do not require direct communication. This feature is very useful in all those scenarios (e.g. disaster scenarios) where wireless communication may be limited or unavailable. The most important feature of the approach is that the computation costs do not increase with the number of robots. Thus, in principle, there is no limit, other than the physical one, to the number of robots composing the team.

\section{EXPERIMENTAL RESULTS}

The coordinated exploration approach has been tested in various simulated environments generated by the National Institute of Standards and Technology (NIST) on the USARSim platform. They provide both indoor and outdoor scenarios of the size bigger than $1000 \mathrm{~m}^{2}$, reconstructing the situation after a real disaster. On these maps, we competed against other teams, during the RoboCup'06 (Homepage of Robocup, 2006) Virtual Robots competition, where our team won the first prize (Kleiner and Ziparo, 2006). In this competition, virtual teams of autonomous or teleoperated robots have to find victims within 20 minutes while exploring an unknown environment. The current version of USARSim is capable of simulating up to 12 robots at the same time. 
Most of the teams applied frontier cell-based exploration on global occupancy grids. In particular: selfish exploration and map merging was used by IUB (Nevatia et al., 2006) and UVA (Pfingsthorn et al., 2006), operator-based frontier selection and task assignment by SPQR, and tele-operation by STEEL (Scerri et al., 2004; Tambe, 1997) and GROK.

\begin{tabular}{ll|llllll} 
& & Rrfr & GROK & IUB & SPQR & STEEL Uva \\
\hline PREl1 & \# Robots & 12 & 1 & 6 & 4 & 6 & 1 \\
& Area [m2] & $\mathbf{9 0 2}$ & 31 & 70 & 197 & 353 & 46 \\
\hline Prel2 & \# Robots & 12 & 1 & 4 & 4 & 6 & 8 \\
& Area [m2] & $\mathbf{5 5 0}$ & 61 & 105 & 191 & 174 & 104 \\
\hline PREL3 & \# Robots & 10 & 1 & 5 & 7 & 6 & 7 \\
& Area [m2] & $\mathbf{3 1 0}$ & 59 & 164 & 44 & 124 & 120 \\
\hline \multirow{2}{*}{ SEMI1 } & \# Robots & 8 & 1 & 6 & 4 & 6 & 6 \\
& Area [m2] & $\mathbf{5 7 9}$ & 27 & 227 & 96 & 134 & 262 \\
\hline \multirow{2}{*}{ SEMI2 } & \# Robots & 8 & 1 & 6 & 5 & 6 & 7 \\
& Area [m2] & $\mathbf{1 2 7 6}$ & 82 & 139 & 123 & 139 & 286 \\
\hline FINAL1 & \# Robots & 8 & - & 8 & - & - & - \\
& Area [m2] & $\mathbf{1 2 0 3}-$ & 210 & - & - & - \\
\hline FInAl2 & \# Robots & 8 & - & 6 & - & - & - \\
& Area [m2] & $\mathbf{3 5 0}$ & - & 136 & - & - & - \\
\hline
\end{tabular}

Table 1. Exploration results from RoboCup '06

Table 6 gives an overview on the number of deployed robots, and area explored by each team. As can clearly be seen, we were able to deploy the largest robot team, while exploring an area bigger up to a magnitude than any other team. Due to the modest computational resources needed by the local approach, we were able to run 12 robots on a single Pentium4, $3 \mathrm{GHz}$.

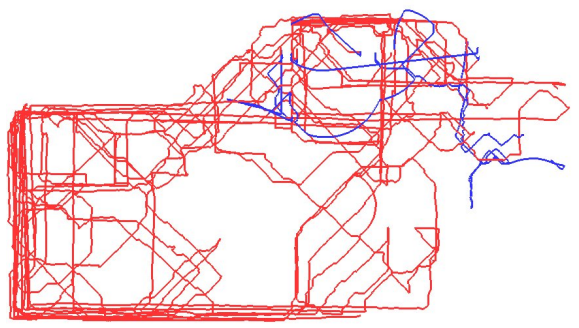

(a)

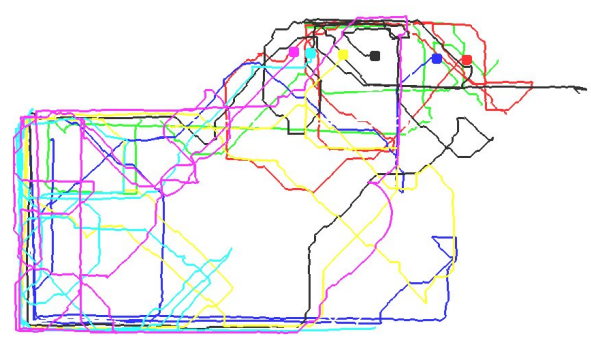

(b)

Fig. 2. Exploration trajectories recorded during the finals: (a) Comparison between our approach (red line) and the other finalist (blue line). (b) Coordinated exploration of our robots, whereas each robot is represent by a different color.

Figure 2(a) depicts the joint trajectory of each team generated during the semi-final and final, whereas (b) shows the single trajectory of each robot of our team on the same map, respectively.
The efficiency of the RFID-based coordination is documented by the differently colored trajectories of each single robot.

We tested the RFID-SLAM approach by collecting data from the USARSim maps from the competitions. The sensor model was simulated accordingly to the model presented in Section 3 . The experiments were conducted over multiple runs, and simulated for four types of environments according to real data (Seidel and Rapport, 1992). Table 2 summarizes the cross-Track error (XTE), measuring the error orthogonal to the true robot path, and the along-Track error (ATE), measuring the error tangential to the path. The column in the table represent four environments, with varying values of the mean path loss exponent $n$ and the standard deviation $\sigma$ for the signal power measurements, which both have been chosen from (Seidel and Rapport, 1992). During the experiment the robot explored an area of approximately $500 \mathrm{~m}^{2}$, driving through heterogeneous surfaces and overcoming small obstacles.

\begin{tabular}{|l|c|c|c|c|}
\hline & B1 & B2 & B3 & B4 \\
\hline XTE mean $[\mathrm{m}]$ & 0.3 & 0.4 & 0.4 & 0.3 \\
\hline XTE $\sigma[\mathrm{m}]$ & 0.2 & 0.2 & 0.3 & 0.2 \\
\hline XTE max $[\mathrm{m}]$ & 1.0 & 1.0 & 1.2 & 0.7 \\
\hline \hline ATE mean $[\mathrm{m}]$ & 0.3 & 0.4 & 0.4 & 0.3 \\
\hline ATE $\sigma[\mathrm{m}]$ & 0.2 & 0.5 & 0.3 & 0.2 \\
\hline ATE max $[\mathrm{m}]$ & 1.1 & 0.9 & 1.3 & 0.8 \\
\hline \hline Cart. mean $[\mathrm{m}]$ & 0.4 & 0.6 & 0.7 & 0.4 \\
\hline Cart. $\sigma[\mathrm{m}]$ & 0.2 & 0.2 & 0.3 & 0.2 \\
\hline Cart. max $[\mathrm{m}]$ & 1.2 & 1.2 & 1.3 & 0.8 \\
\hline
\end{tabular}

Table 2. Cross-Track Error (XTE), Along-Track Error (ATE), and Cartesian Error from EKF-based SLAM with varying model parameters $n$ and $\sigma$. All values are in meters.

\section{DISCUSSION}

In this paper we presented RFID-based exploration and SLAM algorithms for Urban Search and Rescue Robots. The RFID-based approaches allow teams of robots to explore efficiently large areas under severe communication and operational constraints. Experimental results for the exploration where obtained using the USARSim simulator during the RoboCup'07 Virtual Robot Competition. In the competition, our team outperformed other approaches showing that RFIDbased exploration can efficiently and effectively coordinate large teams of robots. Based on the same simulator and scenarios, we performed experiments for the RFID-SLAM approach simulating a realistic sensor model for the RFID reader. The results show that robots, using RFIDs as features, can correct noisy odometry in presence of bumps, obstacles to overcome, and heterogeneous surfaces. RFID features greatly simplify the task of multi-robot SLAM in two ways: i) features can 
be uniquely identified, solving trivially data association problems and ii) the number of features is low w.r.t. visual features and thus the SLAM problem is tractable even for large areas. As future work, we are planning to extend the SLAM approach to omnidirectional antennas (grouping perceptions for triangulation) and to perform experiments on teams of real robots. Moreover, we are investigating how to extend the approach to cooperative SLAM by programming the position estimates of the features in the RFID tags.

\section{REFERENCES}

Balakirsky, S., C. Scrapper, S. Carpin and M. Lewis. (2006). "USARSim: providing a framework for multi-robot performance evaluation".. In: Proceedings of PerMIS 2006.

Balch, T. and R. C. Arkin (1994). Communication in reactive multiagent robotic systems. Autonomous Robots 1(1), 27-52.

Bohn, Jürgen and Friedemann Mattern (2004). Super-distributed rfid tag infrastructures. In: Proceedings of the 2nd European Symposium on Ambient Intelligence (EUSAI 2004). number 3295 In: Lecture Notes in Computer Science (LNCS). Springer-Verlag. Eindhoven, The Netherlands. pp. 1-12. Âl' SpringerVerlag.

CAEN (2007). Homepage of caen rfid. http:// www. caen.it/index.php.

Carpin, S., M. Lewis, J. Wang, S. Balakirsky and C. Scrapper. (2006). Bridging the gap between simulation and reality in urban search and rescue.. In: Robocup 2006: Robot Soccer World Cup X. Springer, LNAI.

Durrant-Whyte, H., D. Rye and E. Nebot (1996). Localisation of automatic guided vehicles. In: Robotics Research: The 7th International Symposium (ISRR'95). Springer Verlag. pp. 613-625.

Hähnel, D., W. Burgard, D. Fox, K. Fishkin and M. Philipose (2004). Mapping and localization with rfid technology. In: Proc. of the IEEE International Conference on Robotics and Automation (ICRA).

Homepage of Robocup (2006). http://www . robocup2006. org.

Kantor, G. and S. Singh (2002). Preliminary results in range-only localization and mapping. In: Proc. of the IEEE International Conference on Robotics and Automation (ICRA). Washington D.C., USA.

Kantor, G., S. Singh, R. Peterson, D. Rus, A. Das, V. Kumar, G. Pereira and J. Spletzer (2003). Distributed search and rescue with robot and sensor team. In: Proceedings of the Fourth International Conference on Field and Service Robotics. Sage Publications. pp. 327-332.

Kehagias, A., J. Djugash and S. Singh (2006). Range-only slam with interpolated range data. Technical Report CMU-RI-TR-06-26.
Robotics Institute, Carnegie Mellon University.

Kleiner, A. and V.A. Ziparo (2006). Homepage of virtual rescuerobots freiburg. http://gkiweb. informatik . uni-freiburg.de/ ${ }^{\text {rescue/virtual/. }}$

Kleiner, A., J. Prediger and B. Nebel (2006). Rfid technology-based exploration and slam for search and rescue. In: Proc. of the IEEE/RSJ Int. Conf. on Intelligent Robots and Systems (IROS).

Kurth, D., G. Kantor and S. Singh (2003). Experimental results in range-only localization with radio. In: Proc. of the IEEE/RSJ Int. Conf. on Intelligent Robots and Systems (IROS). Las Vegas, USA.

Lu, F. and E. Milios (1997). Globally consistent range scan alignment for environment mapping. Auton. Robots 4, 333-349.

Miller, L.E., P. F. Wilson, N. P. Bryner, Francis, J. R. Guerrieri, D. W. Stroup and L. KleinBerndt (2006). Rfid-assisted indoor localization and communication for first responders. In: Proc. of the Int. Symposium on Advanced Radio Technologies.

Nevatia, Yashodan, Mentar Mahmudi, Stefan Markov, Ravi Rathnam, Todor Stoyanov, and Stefano Carpin (2006). Virtual-iub: the 2006 iub virtual robots team. In: Proc. Int. RoboCup Symposium '06. Bremen, Germany.

Pfingsthorn, Max, Bayu Slamet, Arnoud Visser and Nikos Vlassis (2006). Uva rescue team 2006 robocup rescue - simulation league. In: Proc. Int. RoboCup Symposium '06. Bremen, Germany.

Russell, Stuart J. and Peter Norvig (2003). Artificial Intelligence: A Modern Approach. Pearson Education.

Scerri, P., Y. Xu, E. Liao, G. Lai, M. Lewis and K. Sycara (2004). Coordinating large groups of wide area search munitions. In: Recent Developments in Cooperative Control and Optimization (D. Grundel, R. Murphey and P. Pandalos, Eds.). pp. 451-480. Singapore: World Scientific.

Seidel, S. Y. and T. S. Rapport (1992). $914 \mathrm{mhz}$ path loss prediction model for indoor wireless communications in multi-floored buildings. IEEE Trans. on Antennas and Propagation.

Svennebring, J. and S. Koenig (2004). Building terrain-covering ant robots: A feasibility study.. Auton. Robots 16(3), 313-332.

Tambe, M (1997). Towards flexible teamwork. Journal of Artificial Intelligence Research 7, 88-124.

Yamauchi, B. (1997). A frontier-based approach for autonomous exploration. In: IEEE International Symposium on Computational Intelligence in Robotics and Automation (CIRA 'gr).

Ziparo, V.A., A. Kleiner, B. Nebel and D. Nardi (2007). Rfid-based exploration for large robot teams. In: Proc. of the IEEE Int. Conf. on 
Robotics and Automation (ICRA). Rome, Italy. to appear. 